\title{
Does RIFLE score a bullseye with AKI and post-redo CABG outcomes?
}

\author{
George Leslie Hicks, Jr, MD
}

\author{
From the Strong Memorial Department of Surgery, University of Rochester Medical Center, Rochester, NY \\ Disclosures: Author has nothing to disclose with regard to commercial support. \\ Received for publication Feb 24, 2016; accepted for publication Feb 25, 2016; available ahead of print March 16, \\ 2016. \\ Address for reprints: George Leslie Hicks, Jr, MD, Strong Memorial Department of Surgery, University of \\ Rochester Medical Center, Rochester, NY 14642 (E-mail: george_hicks@urmc.rochester.edu). \\ J Thorac Cardiovasc Surg 2016;152:243-4 \\ $0022-5223 / \$ 36.00$ \\ Copyright (C) 2016 Published by Elsevier Inc. on behalf of The American Association for Thoracic Surgery \\ http://dx.doi.org/10.1016/j.jtcvs.2016.02.056
}

The study by Zakkar and colleagues published in this issue of the Journal ${ }^{1}$ is a single-institution retrospective study analyzing more than 15,000 consecutive patients undergoing coronary artery bypass grafting (CABG) over a 16-year period. Among the 380 patients enrolled, 237 $(62.3 \%)$ underwent redo (reoperative) CABG using cardiopulmonary bypass and cardioplegic arrest, and the remaining $143(37.6 \%)$ underwent beating-heart offpump redo CABG. These 380 patients undergoing redo CABG were divided into those with no postoperative evidence of acute kidney injury (AKI) (no-AKI group; $\mathrm{n}=320$ ) and those with AKI (AKI group; $\mathrm{n}=60$ ) using the RIFLE (risk, injury, failure, loss, end-stage renal disease) criteria to document postoperative AKI.

The authors determined the presence of AKI using a patient's baseline serum creatinine $(\mathrm{SCr})$ and estimated glomerular filtration rate (eGFR) values derived from their institutional database registry, with AKI defined by RIFLE criteria as a $50 \%$ change in $\mathrm{SCr}$ postoperatively compared with the baseline value. The authors used Cox regression analyses comparing groups for risk factors associated with $\mathrm{AKI}$, and then used 1:1 propensity matching of 60 patients in each group to analyze health outcomes and survival.

The 60 patients who developed postoperative AKI were grouped into RIFLE categories: $\mathrm{R}, \mathrm{n}=39$ (65\%); $\mathrm{I}, \mathrm{n}=15$ $(25 \%)$; and $\mathrm{F}, \mathrm{n}=6(10 \%)$. Compared with the patients without AKI, they had on average higher incidences of moderate to poor left ventricular ejection fraction, higher angina class, reduced eGFR $(50 \%$ vs $25.6 \% ; P<.01)$, higher SCr levels (mean, $117 \pm 35.9 \mu \mathrm{mol} / \mathrm{L}$ vs $100 \pm 29 \mu \mathrm{mol} / \mathrm{L}$; $P<.01$ ), and higher Euroscore II.

The 30-day mortality was $4.7 \%$, with 18 deaths due to multiorgan failure/sepsis $(\mathrm{n}=11)$, cardiogenic shock $(\mathrm{n}=5)$, or permanent stroke $(\mathrm{n}=2)$. For cause-and-effect considerations, the 18 patients suffering early death also sustained the following postoperative complications: 7 cases of acute renal failure requiring dialysis, 7 cases of cardiogenic shock, 2 cases of permanent stroke, 4 cases of respiratory

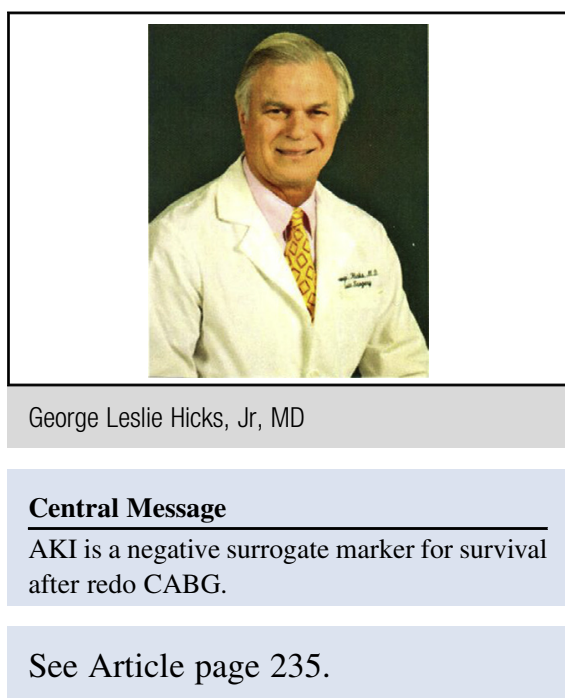

failure requiring tracheostomy, and 1 cases of deep mediastinitis. Univariate analysis showed that AKI, the use of nitrates, Euroscore II, emergency operation, and preoperative use of an intra-aortic balloon pump (IABP) were associated with increased 30-day mortality. Multivariate analysis suggested that that AKI (odds ratio [OR], 3.74; 95\% confidence interval [CI], 1.3-10.5; $P<.01$ ), Euroscore II (OR, 1.27; 95\% CI, 1.07-1.52; $P<.01)$, and preoperative IABP (OR, 6.9; 95\% CI, 2.24-20.3; $P<.01$ ) are independent predictors of 30-day mortality in patients undergoing redoCABG.

The occurrence of in-hospital AKI was associated with increased 30 -day mortality $(13.3 \%$ in the AKI group vs $3.1 \%$ in the non-AKI group; $P<.01$ ), reoperation for bleeding $(6.7 \%$ vs $1.3 \% ; P=.03)$, and prolonged mean hospital length of stay $(13.6 \pm 12.8$ days vs $8.35 \pm 6.4$ days; $P<.01)$.

The median duration of follow-up was 7 years. Overall survival of $93 \%$ after redo CABG was $93 \%$ (80\% in the AKI group vs $93 \%$ in the non-AKI group) at 1 year, $79 \%$ (64\% in the AKI group vs $85 \%$ in the non-AKI group) at 5 years, 64\% (51\% in the AKI group vs $68 \%$ in the non-AKI group) at 10 years. The authors conclude that the presence of AKI as defined by the RIFLE criteria is a powerful predictor of outcome after redo $\mathrm{CABG}$ and may aid decision making and securing of patient consent before CABG.

This paper presents the classic paradox, in that I have to agree to disagree. There is no doubt that the development of AKI postoperatively is a negative prognostic factor, 
although as the authors point out they were unable to provide a suitable comparison group for those undergoing primary CABG operations. Their data and others emphasize the fact that postoperative AKI is most likely a surrogate marker for survival disadvantage, given that the etiology of AKI is multifactorial, representing increased atherosclerotic burden, increased need for blood and inotropic support, and cardiogenic shock, to mention a few factors. The authors report that their AKI group had worse comparative survival than the non-AKI group during the first few years (1-3) postoperatively, but that the 2 groups had parallel curves thereafter (perhaps owing to small numbers). Unfortunately, the data retrieved using their registry system does not provide cause of death and we can only assume that those factors that negatively affected patients early (poor ventricular function, stroke, and perhaps need for dialysis) also had a negative role in the longer term. Moreover, their data do not evaluate relative risk. Are all levels of the RIFLE analysis created equal, or do the R and I levels have better outcomes then level F?

Furthermore, the authors provide no insight into how this negative surrogate (AKI) postoperatively could be reduced and even, owing to small numbers and surgeon preference, whether on-pump or off-pump CABG is most beneficial. It is probably an oversimplification to believe that the RIFLE criteria, which is a postoperative tool, can be used to counsel patients and assess risk preoperatively compared with the more conventional Euroscore II analysis. Thus, the authors went through an exhaustive statistical analysis to determine that postoperative AKI negatively influences patient survival after redo CABG when other, perhaps more influential confounding factors, such as poor ventricular function, need for intra-aortic balloon counterpulsation, increased blood transfusions, higher Euroscore II, and stroke, are present. Therefore, I believe that the authors are correct in their deduction, but I do not feel that RIFLE comes close to the bullseye in establishing this observation.

\section{Reference}

1. Zakkar M, Bruno VD, Guida G, Angelini GD, Chivasso PP, Suleiman MS, et al. Postoperative acute kidney injury defined by risk, injury, failure, loss, and end-stage criteria predicts early health outcome and long-term survival in patients undergoing redo coronary artery bypass graft surgery. J Thorac Cardiovasc Surg. 2016;152: 235-42. 